\title{
Esponjas (Porifera, Demospongiae) da plataforma continental ao largo do Estado do Amapá, Brasil
}

\author{
Beatriz Mothes ${ }^{1}$; Maurício A. de Campos $^{2}$; Cléa B. Lerner ${ }^{1} \&$ Carla M. M. da Silva ${ }^{3}$ \\ ${ }^{1}$ Núcleo de Invertebrados, Museu de Ciências Naturais, Fundação Zoobotânica do Rio Grande do Sul. Rua Salvador França \\ 1427, Jardim Botânico, 90690-000 Porto Alegre, Rio Grande do Sul, Brasil. E-mail: bmothes@fzb.rs.gov.br \\ 2 Programa de Pós Graduação em Biologia Animal, Universidade Federal do Rio Grande do Sul. Avenida Bento Gonçalves \\ 9500, Prédio 43435, Campus do Vale, 91501-970 Porto Alegre, Rio Grande do Sul, Brasil. \\ ${ }^{3}$ Laboratório de Biologia de Porifera, Departamento de Zoologia, Instituto de Biologia, Universidade Federal da Bahia. Rua \\ Barão de Geremoabo, 40170-290 Salvador, Bahia, Brasil.
}

\begin{abstract}
Sponges (Porifera, Demospongiae) of the continental shelf off the coast of Amapá State, Brazil. The collections were dredged off the coast $\left(04^{\circ} 13^{\prime} 00^{\prime \prime}-02^{\circ} 34^{\prime} 02^{\prime \prime} \mathrm{N}, 47^{\circ} 54^{\prime} 05^{\prime \prime}-50^{\circ} 50^{\prime} 07^{\prime \prime} \mathrm{W}\right)$. They were collected between 56 to 95 depths, during an oceanographic expedition, Diretoria de Hidrografia e Navegação da Marinha do Brasil, by R/V “Almirante Saldanha” in 1968. Eight species are here registered, among which Niphates alba Van Soest, 1980, Placospongia melobesioides Gray, 1867 and Terpios belindae Rützler \& Smith, 1993 represent new registers for Brazil. The other species: Aplysina fulva (Pallas, 1766), Ircinia strobilina (Lamarck, 1816), Niphates erecta Duchassaing \& Michelotti, 1864, Neopetrosia proxima (Duchassaing \& Michelotti, 1864) and Plakinastrella onkodes Uliczka, 1929 represent new records to the studied area. All registered species are also known from Caribbean. Specimens are deposited in the Porifera Collection of the Museu de Ciências Naturais, Fundação Zoobotânica do Rio Grande do Sul, Brazil.

KEY WORDS. Continental shelf; north Brazilian coast; sponge distribution; taxonomy.
\end{abstract}

RESUMO. As amostras foram coletadas com draga retangular ao largo da costa $\left(04^{\circ} 13^{\prime} 00^{\prime \prime}-02^{\circ} 34^{\prime} 02^{\prime \prime} \mathrm{N}, 47^{\circ} 54^{\prime} 05^{\prime \prime}\right.$ 5050’07"W), pela Comissão Pesca Norte I, em 1968, através da Diretoria de Hidrografia e Navegação da Marinha do Brasil com o N./Oc. "Almirante Saldanha" em profundidades que variaram de 56 a $95 \mathrm{~m}$. Oito espécies são aqui registradas, entre as quais Niphates alba Van Soest, 1980, Placospongia melobesioides Gray, 1867 e Terpios belindae Rützler \& Smith, 1993 são identificadas pela primeira vez para a costa brasileira. As demais espécies, Aplysina fulva (Pallas, 1766), Ircinia strobilina (Lamarck, 1816), Niphates erecta (Duchassaing \& Michelotti, 1864), Neopetrosia proxima (Duchassaing \& Michelotti, 1864) e Plakinastrella onkodes Uliczka, 1929 são conhecidas pela primeira vez para a área estudada. Todas as espécies identificadas são também registradas para o Caribe. Os espécimes encontram-se depositados na Coleção de Porifera do Museu de Ciências Naturais, Fundação Zoobotânica do Rio Grande do Sul, Brasil.

PALAVRAS-CHAVE. Costa norte brasileira; distribuição de esponjas; plataforma continental; taxonomia.

A costa do Amapá recebe a influência da corrente das Guianas, originária de uma bifurcação da Corrente Equatorial Sul, vinda do Golfo da Guiné (África), fluindo sentido noroeste ao longo da costa da América do Sul, caraterizando-se também por águas quentes e salinidade relativamente alta (ЕкмAN 1953). A descarga de dois grandes rios equatoriais nesta região, o Amazonas e o Orinoco, são responsáveis pela ausência de um substrato firme e diminuição de salinidade em regiões próximas ao litoral (VANNUCCI 1964).

Apenas três espécies de Demospongiae estão registradas para a área estudada: Mycale quadripartita Boury-Esnault, 1973 (HAJDU \& Desqueyroux-Faundez 1994), Biemna microacanthosigma Mothes, Hajdu, Lerner \& Soest, 2004 (Mothes et al. 2004b) e Biemna trisigmata Mothes \& Campos, 2004 (Mотнеs \& CAMpos 2004).

Objetiva-se na presente pesquisa levar a efeito a identificação taxonômica dos espécimes de Demospongiae da plataforma continental coletados pela Diretoria de Hidrografia e Navegação da Marinha (DHNM) em 1968, entre Cabo Orange e Cabo Norte, além de ampliar o conhecimento de riqueza de esponjas na costa brasileira. 


\section{MATERIAL E MÉTODOS}

O material estudado foi coletado com draga retangular, em profundidades que variaram de 56 a 95 m, pela Comissão Pesca Norte I, em 1968, através da Diretoria de Hidrografia e Navegação da Marinha do Brasil, com o navio oceanográfico "Almirante Saldanha". A área amostrada situa-se entre as coordenadas $04^{\circ} 13^{\prime} 00^{\prime \prime}-02^{\circ} 34^{\prime} 02^{\prime \prime} \mathrm{N}, 47^{\circ} 54^{\prime} 05^{\prime \prime}-50^{\circ} 50^{\prime} 07^{\prime \prime} \mathrm{W}$, ao largo da costa do Estado do Amapá (Fig. 1).

Os espécimes estão depositados na Coleção de Poríferos Marinhos do Museu de Ciências Naturais (MCNPOR) da Fundação Zoobotânica do Rio Grande do Sul. O material é proveniente do Departamento de Oceanografia da UFPE, transferido para o MCN após avaliação prévia daquela Coleção de Porifera, realizada por C. Volkmer Ribeiro com auxílio concedido pelo CNPq.

Metodologia utilizada: preparação de dissociação espicular conforme Mothes-De-Moraes (1978); observação da estrutura esqueletal segue Mothes et al. (2004a) e preparação de stubs de acordo com Mothes \& Lerner (2001). Para o estudo de fibras na Ordem Verongida procedeu-se a retirada de um pedaço da esponja, colocando-o em frasco contendo água sanitária, objetivando a obtenção do retículo de espongina isolado. A seguir as fibras de espongina foram colocadas sobre lâmina, deixando-as secar sob lâmpada incandescente, sendo cobertas com Entellan ${ }^{\circledR}$ e lamínula. Mensurações de escleras e filamentos de espongina são referidas para mínima-média-máxima, $\mathrm{n}=50$ exceto quando indicado. Mensurações de fibras e malhas são referidas para mínima e máxima, (n) indicado no texto. Largura referida após a barra (/). Diagnose dos táxons segue HoOper \& VAN SOEST (2002).

Siglas utilizadas no texto: (MCNPOR) Coleção de Porifera do Museu de Ciências Naturais da Fundação Zoobotânica do Rio Grande do Sul, Porto Alegre, Brasil; (N./Oc.) Navio Oceanográfico; (ZMAPOR) Instituut voor Systematiek en Populatiebiologie (Zoölogisch Museum), Amsterdam, Holanda; (ZMB) Zoologisches Museum für naturkunde an der Universität Humboldt zu Berlin, Berlin, Alemanha.

\section{RESULTADOS Dictyoceratida Irciniidae Gray, 1867 Ircinia strobilina (Lamarck, 1816)} Figs 2-4

Spongia strobilina Lamarck, 1816: 383 (Localidade-tipo ? Mediterrâneo).

Ircinia strobilina; Boury-Esnault, 1973: 289; Muricy \& Moraes, 1998: 216.

Para sinônimos adicionais veja-se WiedenMaYer (1977) e ZEA (1987).

Material examinado. MCNPOR $1896\left(02^{\circ} 57^{\prime} 00^{\prime \prime} \mathrm{N} / 49^{\circ} 04^{\prime}\right.$ $00 ” \mathrm{~W}$ ), profundidade $76 \mathrm{~m}$, substrato biodetrítico.

Material estudado para comparação. MCNPOR 2481 Ircinia strobilina (Lamarck, 1816) [det. Klaus Rützler].

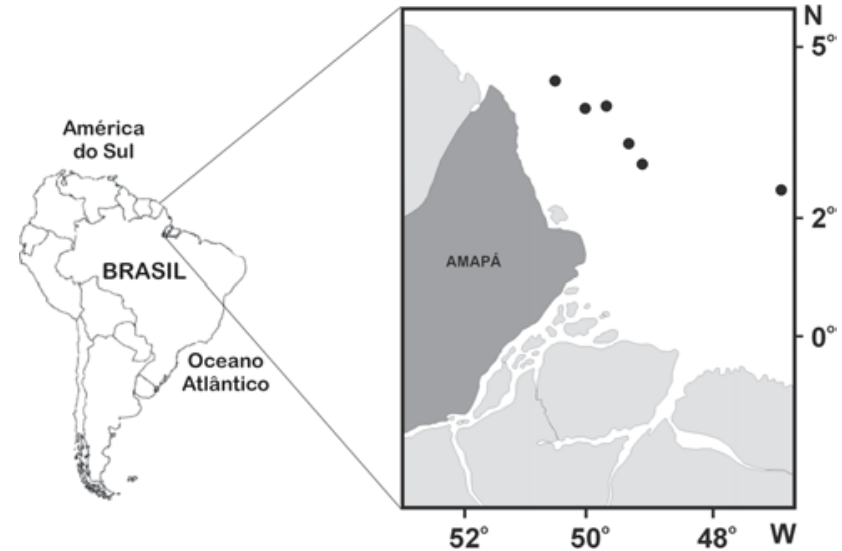

Figura 1. Localização da área. Locais de coleta indicados pelos pontos.

Descrição. Espécime (comprimento 6,0 cm, largura 5,0 cm e altura 3,5 cm) discretamente globular (Fig. 2). Superfície conulosa, de cada cônulo (altura 0,1-0,3 cm; distância entre os cônulos 0,3-0,8 cm) protraem discretamente os ósculos (diâmetro 0,1-0,8 cm) distribuídos em depressões por entre os cônulos; contornando a abertura oscular observa-se uma estrutura anelar; poros não observados. Material preservado de consistência rígida; coloração cinza-rosada externamente e marrom avermelhada internamente.

Esqueleto. No material preservado não se observou distinção entre ectossoma e coanossoma. No interior da esponja ocorre grande quantidade de filamentos de espongina (Fig. 3) (diâmetro 1,1-4,6 $\mu \mathrm{m}$ ), com cabeça esférica ou ovóide (diâmetro 4,0-12 $\mu \mathrm{m}$ ). Observados sem muita nitidez fascículos de fibras primárias ascendentes (Fig. 4) (largura: 560-900 $\mu \mathrm{m}, \mathrm{n}=$ 06), até a superfície e nesta constituindo os cônulos; fibras secundárias muito finas (largura 40-140 $\mu \mathrm{m}, \mathrm{n}=06$ ), conectantes transversalmente às primárias.

Comentários. LAMARCK (1816) registrou como incerta a presença de I. strobilina no Mediterrâneo. A espécie foi identificada no Caribe por Laubenfels (1936), que a redescreveu. Hechtel (1965), ao citá-la para a Jamaica, considerou duvidosa a coespecificidade dos espécimes caribenhos com os da região Indo-Pacífica, alertando para a identificação de I. strobilina por SARÀ (1958), que na verdade poderia tratar-se de I. muscarum conforme VACELET (1959). Mesmo na incerteza de sua localidade-tipo, acredita-se que os espécimes identificados como $I$. strobilina, pertinentes ao Atlântico Ocidental Tropical, sejam diferentes daqueles registrados para o Mediterrâneo e região Indo-Pacífica, por tratar-se de distribuição disjunta.

Distribuição geográfica. Bermudas até Guianas (ZEA 1987); Brasil: Amapá (no presente estudo); ao largo da desembocadura do Rio Amazonas $\left[01^{\circ} 42^{\prime} 00^{\prime \prime}-01^{\circ} 40^{\prime} 00^{\prime \prime} \mathrm{N}, 47^{\circ} 55^{\prime} 00^{\prime \prime}-47^{\circ} 24^{\prime}\right.$ $00^{\prime \prime} \mathrm{W}$, registro de ocorrência e coordenadas fornecido in litt. por Klaus Rützler]; Ceará (Johnson 1971); Pernambuco (Hechtel 1976, Muricy \& Moraes 1998); Bahia (Boury-Esnault 1973).

Revista Brasileira de Zoologia 23 (3): 667-677, setembro, 2006 

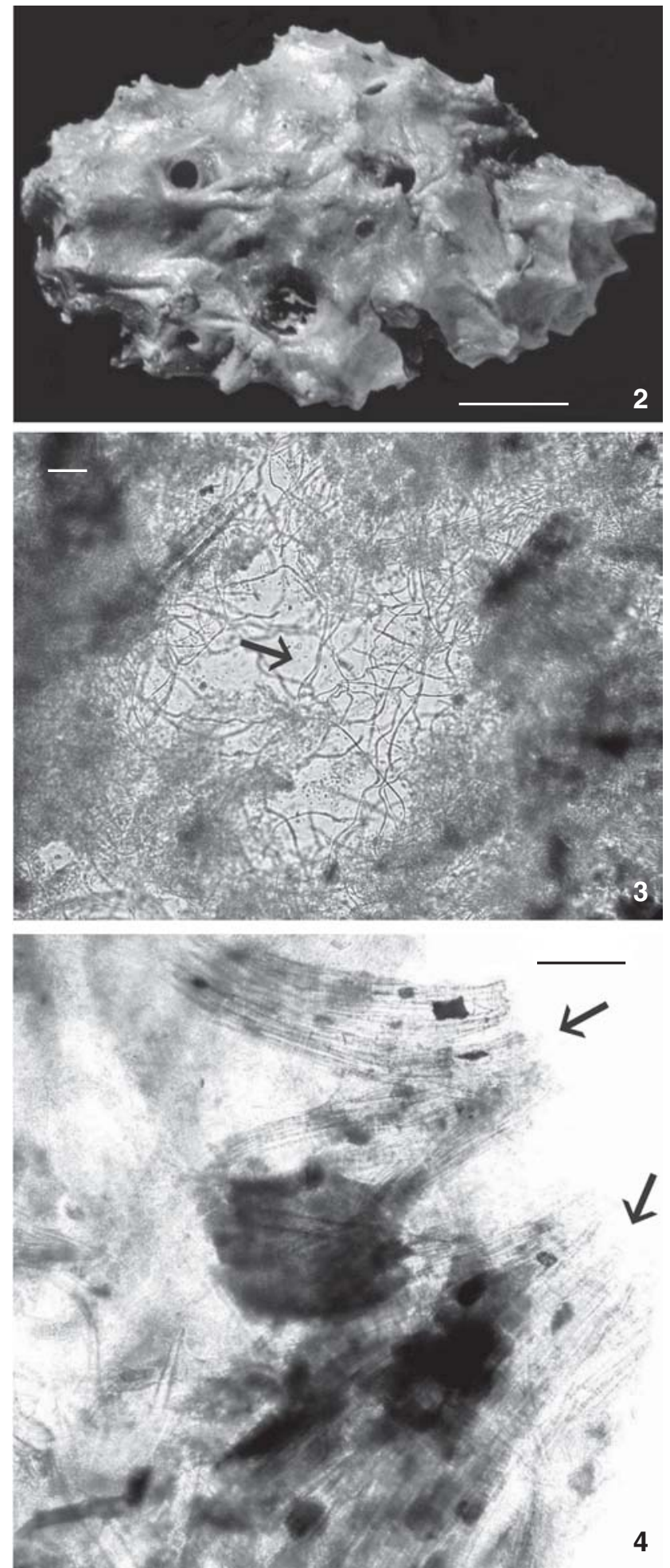

Figuras 2-4. Ircinia strobilina: (2) vista do espécime; (3) filamentos de espongina; (4) fibras de espongina. Escalas: (2) $1 \mathrm{~cm}$; (3-4) $150 \mu \mathrm{m}$.
Distribuição batimétrica. Desde 1 m, Cartagena (ZEA 1987) até $76 \mathrm{~m}$ (presente estudo).

\section{Verongida \\ Aplysinidae Carter, 1875 Aplysina fulva (Pallas, 1766)}

Spongia fulva Pallas, 1766: 383. (Localidade-tipo: "Mare Americanum").

Aplysina fulva; Muricy et al., 1991: 1187; Mothes \& Bastian, 1993: 24, fig. 48; Mothes \& Lerner, 1994: 57; Pinheiro \& Hajdu, 2001: 149, figs 7-15, tab. II.

Para sinônimos adicionais veja Wiedenmayer (1977), Van Soest (1978) e Zea (1987).

Material examinado. MCNPOR $1871\left(02^{\circ} 34^{\prime} 02^{\prime \prime} \mathrm{N} / 47^{\circ} 54^{\prime}\right.$ $05 " \mathrm{~W})$, profundidade $78 \mathrm{~m}$, substrato arenoso/biodetrítico

Material estudado para comparação. ZMAPOR 3704 Aplysina fulva (Pallas, 1766) [det. Rob Van Soest].

Descrição. Fragmento (comprimento 0,5 cm, largura 3,0 cm e altura $4,0 \mathrm{~cm}$ ) com duas discretas projeções arredondadas. Superfície microconulosa (distância entre os cônulos $0,1 \mathrm{~cm}$ ), cônulos pontiagudos (altura $0,1 \mathrm{~cm}$ ). Ósculos e poros não observados. Material preservado de consistência pouco compressível e coloração marrom amarelada.

Esqueleto. Descrição de acordo com PinHeIro \& Hajdu (2001). Dimensões: fibras 47,5-142 $\mu \mathrm{m}$ de largura, medula ocupando12$60 \%$ da fibra e malhas com 475-1900/304-975 $\mu \mathrm{m}$ ( $\mathrm{n}=08)$.

Distribuição geográfica. Bahamas até Curaçao (WIEDENMAYER 1977, VAN SoEsT 1978, ZeA 1987); Brasil: Amapá (no presente estudo); ao largo da desembocadura do Rio Amazonas [01\% $42^{\prime} 00^{\prime \prime} S$ $47^{\circ} 24^{\prime} 00^{\prime \prime}$, registro de ocorrência e coordenadas fornecidas in litt. por Klaus Rützler]; Ceará (JoHnson 1971); Arquipélago de Fernando de Noronha, Ilha Rata (Mothes \& BASTIAN 1993); Espírito Santo, Guarapari (Solé-Cava et al. 1981); Rio de Janeiro, Arraial do Cabo (Muricy et al. 1991, 1993); São Paulo, canal de São Sebastião (PINHeIro \& Hajdu 2001); Bombinhas, Santa Catarina (MOTHes \& Lerner 1994).

Distribuição batimétrica. Desde $1 \mathrm{~m}$, Canal de São Sebastião, São Paulo (PINHeiro \& Hajdu 2001) até 100 m, Barbados (VAN SOEST 1978).

\section{Haplosclerida Haplosclerina Niphatidae Van Soest, 1980} Niphates erecta Duchassaing \& Michelotti, 1864

Niphates erecta Duchassaing \& Michelotti, 1864: 93, pl. XXI, fig. 3. (Localidade-tipo: St. Thomas).

Para sinônimos adicionais veja CAMPos et al. (2005).

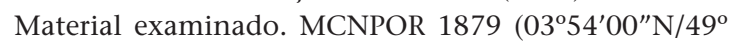
$\left.45^{\prime} 00^{\prime \prime} \mathrm{W}\right)$, profundidade $95 \mathrm{~m}$, substrato detrítico, 26.IX.1968; MCNPOR $1881\left(02^{\circ} 34^{\prime} 02^{\prime \prime} \mathrm{N} / 47^{\circ} 54^{\prime} 05^{\prime \prime} \mathrm{W}\right)$, profundidade $95 \mathrm{~m}$, sem registro de substrato, 24.IX.1968. 
Descrição. Amostras fragmentadas (fragmento maior: $\mathrm{MCN}$ 1881, comprimento $4,0 \mathrm{~cm}$, espessura $0,2 \mathrm{~cm}$ ). Superfície discretamente híspida. Ósculos (diâmetro 0,2-0,3 cm) dispersos pela superfície. Material preservado de consistência dura, porém friável e coloração bege clara (MCN 1879) e cinza (MCN 1881).

Esqueleto. Descrição de acordo com CAmpos et al. (2005). Dimensões. Ectossoma: fibras (diâmetro 37,5-137,5 $\mu \mathrm{m}, \mathrm{n}=20$ ), malhas (diâmetro 180-450 $\mu \mathrm{m}, \mathrm{n}=15$ ); coanossoma: fibras primárias multiespiculares (diâmetro 40-87,5 $\mu \mathrm{m}, \mathrm{n}=15$ ), fibras secundárias pauciespiculares, 2-7 escleras (diâmetro 20-40 m, $\mathrm{n}=15$ ), malhas poligonais (diâmetro $80450 \mu \mathrm{m}, \mathrm{n}=15$ ).

Escleras. Megascleras: óxeas, geralmente encurvadas, algumas fusiformes, extremidades arredondadas ou gradualmente pontiagudas, dimensões: 161,5-198,2-218,5/3,5-6,5-9,2 $\mu \mathrm{m}$ (MCN 1881) e 100-197,4-270/3,8-8, 5-12,5 um (MCN 1879). Microscleras ausentes.

Distribuição geográfica. Bermudas a Venezuela (WIEDENMAYER 1977; VAN Soest 1980, ZeA 1987); Brasil: Amapá (no presente estudo); Maranhão (Campos et al. 2005); Pernambuco (Hechtel 1976).

Distribuição batimétrica. Desde 0,1 m, Bonaire (VAN SOEST 1980) até $115 \mathrm{~m}$ (no presente estudo).

\section{Niphates alba Van Soest, 1980}

\section{Figs 5-12}

Niphates alba Van Soest, 1980: 40, fig. 14, pl. VI, fig. 4, pl.VII, fig.1. (Localidade-tipo: Porto Rico); Zea, 1987: 90, fig. 23.

Material examinado. MCNPOR 1889 (0316'00” N-49¹5' $05^{\prime \prime} \mathrm{W}$ ), profundidade $73 \mathrm{~m}$, substrato arenoso/detrítico, 26.IX. 1968.

Material estudado para comparação. Holótipo: ZMAPOR 3942, Porto Rico, Caribe.

Descrição. Fragmento de um ramo (comprimento $4,0 \mathrm{~cm}$ e diâmetro 3,0 cm) (Fig. 5). Superfície microhíspida. Ósculos não observados. Material fixado de consistência compressível e coloração bege.

Esqueleto. Ectossoma com fibras multiespiculares (diâmetro 40-140 $\mu \mathrm{m}, \mathrm{n}=15$ ) (Fig. 6), subdivididas em fibras menores (diâmetro 15-37,5 $\mu \mathrm{m}, \mathrm{n}=15$ ) com no máximo 05 escleras por secção, formando malhas (diâmetro $160-450 \mu \mathrm{m}, \mathrm{n}=15$ ) poligonais. Coanossoma com fibras primárias multiespiculares (diâmetro 7,6-95 $\mu \mathrm{m}, \mathrm{n}=12$ ) (Fig. 7), com disposição discretamente plumosa próxima à superfície, conectadas por fibras secundárias pauciespiculares (diâmetro 3,8-66,5 $\mu \mathrm{m}, \mathrm{n}=12$ ), produzindo malhas retangulares a poligonais (diâmetro150-420 $\mu \mathrm{m}, \mathrm{n}=12$ ).

Escleras. Megascleras: estrôngilos - alguns retos (Figs 8, 11), ou com discreta curvatura (Fig. 9); raras óxeas (Fig. 10), gradualmente aguçadas (Fig. 12); dimensões: 190-228-247/6,09,2-11,5 $\mu \mathrm{m}$. Microscleras ausentes.

Distribuição geográfica. Porto Rico e Colômbia (ZEA 1987); Brasil: Amapá (no presente estudo).

Distribuição batimétrica. Desde 0,1 m, Bonaire (VAN SOEST 1980) até $73 \mathrm{~m}$ (no presente estudo).

\section{Petrosina \\ Petrosiidae van Soest, 1980 \\ Neopetrosia proxima (Duchassaing \& Michelotti, 1864)}

Thalysias proxima Duchassaing \& Michelotti, 1864: 84, pl. VIII, figs 2-3. (Localidade-tipo: Porto Rico)

Para sinônimos adicionais veja CAMPos et al. (2005).

Material examinado. MCNPOR $1888\left(04^{\circ} 13^{\prime} 00^{\prime \prime} \mathrm{N}, 50^{\circ} 31^{\prime}\right.$ $\left.00^{\prime \prime} \mathrm{W}\right)$, profundidade $76 \mathrm{~m}$, substrato arenoso/detrítico, 27.IX. 1968; MCN 1899 (02 $\left.57^{\prime} 00^{\prime \prime} \mathrm{N}, 49^{\circ} 04^{\prime} 00^{\prime \prime} \mathrm{W}\right)$, profundidade 73$76 \mathrm{~m}$, sem registro de substrato, 24.XI.1968.

Descrição. Fragmentos cilíndricos. Fragmento maior (MCN 1899: altura 2,5 cm e diâmetro 1,5 cm). Superfície lisa. Ósculos não observados. Material preservado de consistência dura, quando seco semelhante à pedra pomes; coloração cinza clara (MCN 1888) e discretamente rosada (MCN 1899).

Esqueleto. Descrição de acordo com CAMpos et al. (2005). Dimensões: ectossoma: fibras (diâmetro 23-156,4 $\mu \mathrm{m}, \mathrm{n}=20$ ), malhas (diâmetro 104,5-380 $\mu \mathrm{m}, \mathrm{n}=20$ ); coanossoma: canais superficiais (diâmetro $114-456 \mu \mathrm{m}, \mathrm{n}=15$ ), fascículos (diâmetro 171-503,5 $\mu \mathrm{m}, \mathrm{n}=05$ ), fibras primárias (diâmetro 76-218,5 $\mu \mathrm{m}, \mathrm{n}=10$ ) e fibras secundárias (diâmetro 33-85,5 $\mu \mathrm{m}, \mathrm{n}=10$ ).

Escleras. Megascleras: óxeas encurvadas, abruptamente pontiagudas, extremidades telescópicas; dimensões: 161,5183,7-209/2,3-6,6-10,35 um (MCNPOR 1899) e 133-166,8199,5/2,3-5,45-6,9 $\mu \mathrm{m}$ (MCNPOR 1888).

Distribuição geográfica. Porto Rico a Colômbia (ZEA 1987, Van Soest \& Stentoft 1988, Lehnert \& Van Soest 1996); Brasil: Amapá (no presente estudo); ao largo da desembocadura do Rio Amazonas $\left[01^{\circ} 40^{\prime} \mathrm{N}, 47^{\circ} 55^{\prime \prime} \mathrm{W}\right.$, registro de ocorrência e coordenadas fornecidas in litt. por Klaus Rützler] como Xestospongia sp.; Maranhão (CAMPos et al. 2005).

Distribuição batimétrica. Desde $6 \mathrm{~m}$, Bahia de Nenguange, Santa Marta, Colômbia (Zea 1987) a 153 m ao largo de Paynes Bay, Barbados (VAn Soest \& Stentoft 1988).

\section{Hadromerida Placospongiidae Gray, 1867 Placospongia melobesioides Gray, 1867 Figs 13-22}

Placospongia melobesioides Gray, 1867: 128, figs 1-4. (Localidade-tipo: Bornéu, Indo-Pacífico); Vosmaer \& Vernhout, 1902: 9; Rützler, 2002: 198, fíg. 3 A-G, fíg. 4 A-E. Para sinônimos adicionais veja RÜTZLER (2002).

Material examinado. MCNPOR $1874\left(02^{\circ} 57^{\prime} 00^{\prime \prime} \mathrm{N}, 4^{\circ} 04^{\prime}\right.$ $00^{\prime \prime} \mathrm{W}$ ), profundidade $76 \mathrm{~m}$, substrato arenoso/detrítico, 24.IX. 1968.

Material estudado para comparação. ZMAPOR sem registro Placospongia melobesioides [det. B. Alvarez].

Descrição. Fragmento discretamente cônico constituído por duas placas corticais discretamente achatadas, de contorno poligonal, unidas por um discreto sulco periférico (Fig. 13). 

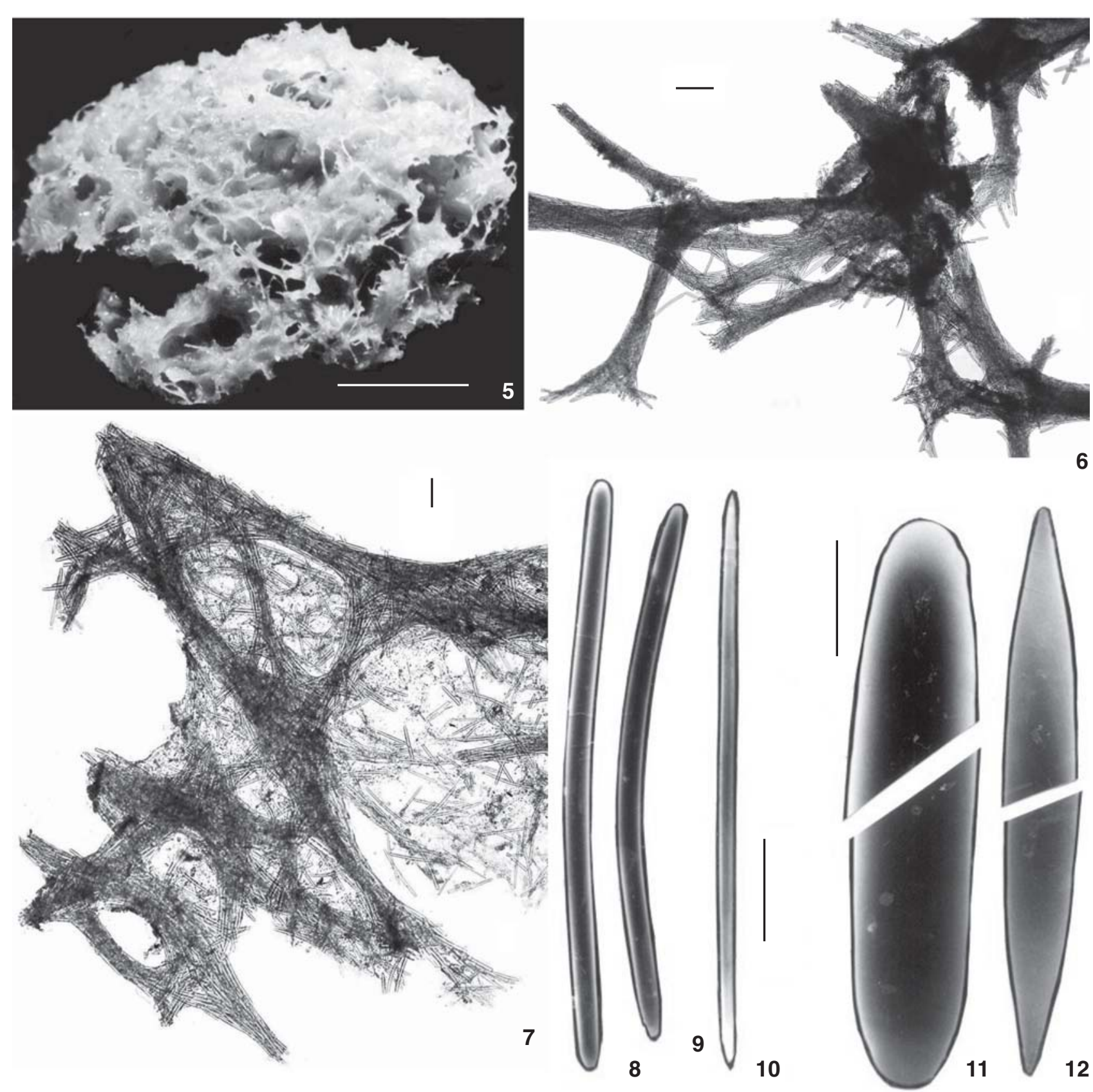

Figuras 5-12. Niphates alba: (5) vista do espécime; (6) ectossoma; (7) coanossoma (8); estrôngilo reto; (9) estrôngilo encurvado; (10) óxea (11) extremidades do estrôngilo; (12) extremidades da óxea. Escalas: (5) 1 cm; (6-7) $300 \mu \mathrm{m}$; (8-10) $50 \mu \mathrm{m}$; (11-12) $10 \mu \mathrm{m}$.

Dimensões da maior placa: espessura $0,3 \mathrm{~cm}$, superfície $0,6 \mathrm{~cm}$. Ósculos e poros não observados. Material preservado de consistência dura na superfície e na porção inferior compressível; coloração da placa marrom escura na porção superior e marrom claro na porção inferior.

Esqueleto. Ectossoma constituído por um córtex (Fig. 14), com uma densa agregação de selenásteres adultos e em vários estágios de desenvolvimento, os quais se estendem também pelo interior do coanossoma; na porção mais superior, ocorrem abundantemente formas semelhantes à espirásteres (= estágios iniciais de selenásteres) (Fig. 15). Por tratar-se de um fragmento muito pequeno não foi possível distinguir a exata locali-

Revista Brasileira de Zoologia 23 (3): 667-677, setembro, 2006 

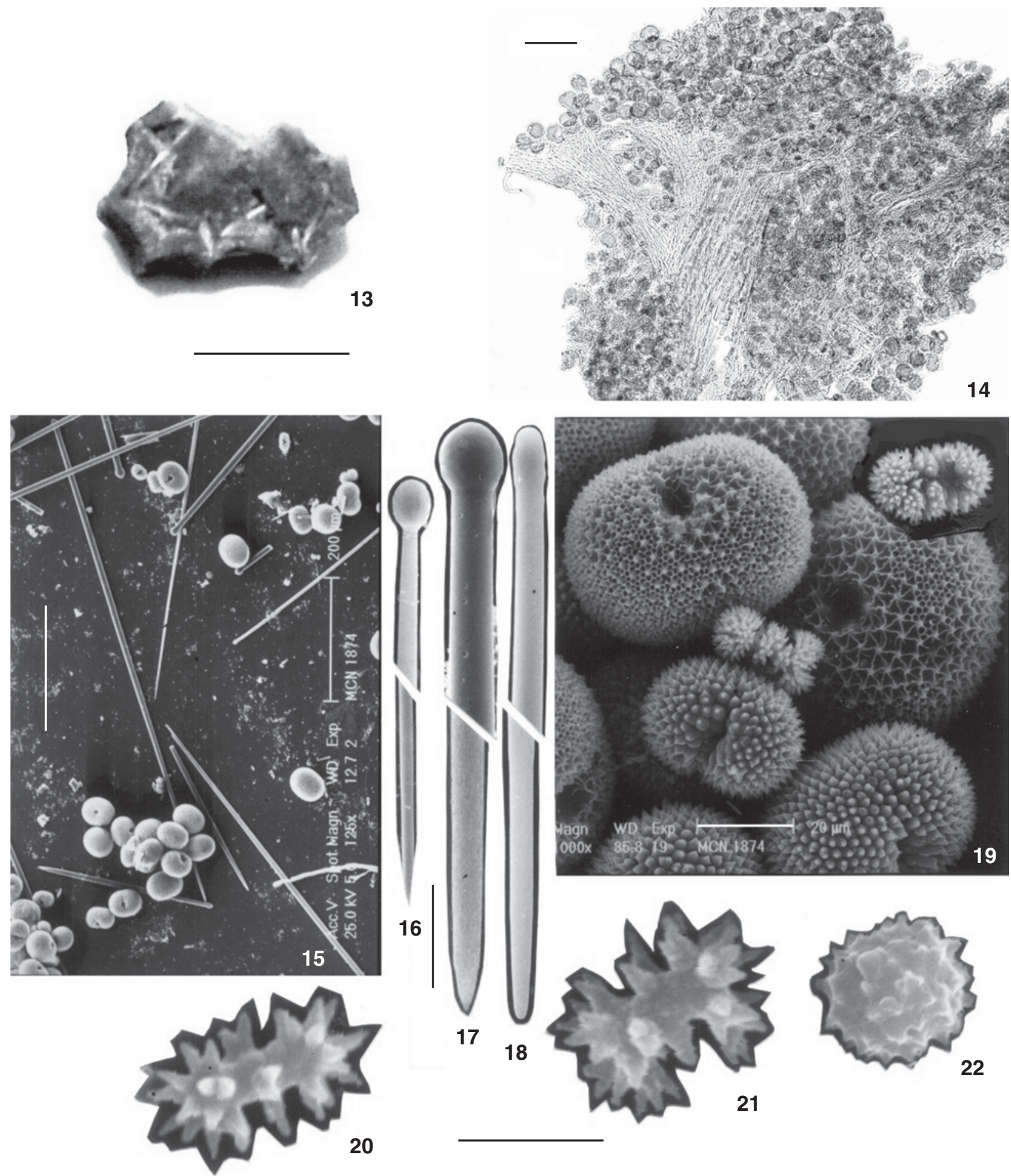

Figuras 13-22. Placospongia melobesioides: (13) vista da placa cortical; (14) esqueleto; (15) conjunto espicular; (16) tilóstilo cortical; (17) tilóstilo coanossomal, cabeça redonda; (18) tilóstilo coanossomal, cabeça bilobada; (19) selenásteres, estágios de desenvolvimento; (20) selenáster, estágio inicial, eixo reto; (21) selenáster, estágio inicial, eixo discretamente espiralado; (22) microesferáter. Escalas: (13) 0,5 cm; (14-15) $150 \mu \mathrm{m}$; (16-19) $20 \mu \mathrm{m}$; (20-22) $2 \mu \mathrm{m}$.

Revista Brasileira de Zoologia 23 (3): 667-677, setembro, 2006 
zação dos pequenos tilóstilos, os quais de acordo com Vosmaer \& Vernhout (1902) estariam dispostos na região periférica dos sulcos, formados entre as placas corticais. Coanossoma com feixes ascendentes de grandes tilóstilos que chegam até a superfície.

Escleras. Megascleras: tilóstilos corticais (Fig. 15) retos, cabeça arredondada, extremidade apical gradualmente pontiaguda (Fig. 16), dimensões: 200-283-360/2,5-6,4-8,8 $\mu \mathrm{m}$, cabeça 3,88,1-10 $\mu \mathrm{m}$; tilóstilos coanossomais retos, cabeça arredondada (Fig. 17) ou algumas vezes bilobada (Fig. 18), extremidade apical gradualmente estrongiliforme ou algumas vezes discretamente mucronada, dimensões: 640-772,2-930/7,5-9,6-11,3 $\mu \mathrm{m}$, cabeça 10-12,8-15 $\mu \mathrm{m}$; selenásteres (= esterrospira Vosmaer \& Vernhout 1902) - com vários estágios de desenvolvimento (Fig. 19), forma ovóide (estágio adulto), dimensões: 77-90-111/26- $\underline{36}-48 \mu \mathrm{m}$, estágios iniciais de selenásteres com eixo reto (Fig. 20) ou discretamente espiralado (Fig. 21), dimensões $>1 \mu \mathrm{m}$; microesferáster observado apenas um (Fig. 22), dimensão $>1 \mu \mathrm{m}$.

Comentários. Conforme Vosmaer \& Vernhout (1902), e corroborado por RÜTZLER (2002), existem apenas três espécies válidas, sendo que destas, $P$. melobesioides Gray, 1867 e $P$. carinata (Bowerbank, 1858) tem registros também para o Caribe.

Tanto $P$. melobesioides como $P$. carinata foram descritas para a região do Indo-Pacífico, sendo que a primeira para Bornéu e posteriormente referida para Curaçao por ARNDT (1927) e a segunda para "South Seas" por Bowerbank (1858), e citada também para a Jamaica por Hechtel (1965). A presença de possíveis linhagens similares trans-istmo, de espécies em ambos os lados do canal do Panamá, é abordada por Laubenfels (1936) e Nichols \& BARNES (2005). Na amostra estudada os espirásteres observados foram considerados como estágios iniciais de desenvolvimento de selenásteres e não espirásteres típicos como os encontrados em $P$. carinata, por esta evidência e pela presença de microesferásteres a amostra foi identificada como P. melobesioides (identificação corroborada por Klaus Rützler, in litt.).

Distribuição geográfica. Bornéu (Gray 1867); Golfo do México (Rützler 2002); Curaçao (Arndt 1927); Brasil: Amapá (no presente estudo); Pernambuco (Boury-Esnault 1973 como $P$. cristata).

Distribuição batimétrica. Desde $25 \mathrm{~m}$, Pernambuco (BourYESNAULT 1973) até $76 \mathrm{~m}$ (no presente estudo).

\section{Suberitidae Schmidt,1870 Terpios belindae Rützler \& Smith, 1993 \\ Figs 23-28}

Terpios belindae Rützler \& Smith, 1993: 338, figs 5, 6, 9, tab. I. (Localidade-tipo: Tobago, Caribe).

Material examinado. MCNPOR $1868\left(02^{\circ} 34^{\prime} 02^{\prime \prime} \mathrm{N}-\right.$ $\left.47^{\circ} 54^{\prime} 05^{\prime \prime} \mathrm{W}\right)$, profundidade $56 \mathrm{~m}$, substrato biodetrítico, 24.IX.1968.

Descrição. Fina película transparente (espessura 0,1-0,2 cm e comprimento $2,5 \mathrm{~cm}$ ) (Fig. 23), incrustante sobre Ectoprocta e fragmento de esponja Calcarea. Superfície bastante híspida.
Ósculos e poros não observados. Material preservado de consistência esponjosa e coloração marrom-clara.

Esqueleto. Ectossoma não diferenciado do coanossoma (Fig. 24). Coanossoma com tilóstilos dispostos em discretos feixes ascendentes, os quais se abrem em forma de tufos na superfície da esponja, entre os feixes ocorrem escleras dispostas de modo confuso. Escleras. Megascleras: tilóstilos - retos, extremidade basal dotada de lóbulos (Fig. 25), extremidade apical gradualmente pontiaguda (Fig. 26). Porção basal pode apresentar-se bilobada (Fig. 27), embora rara, ou tetralobada (Fig. 28) na sua maioria; porção superior dos lóbulos achatada;

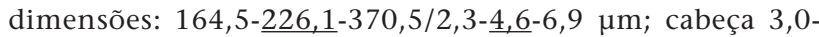
$\underline{4,5} \mu 7,0 / 5,0-\underline{7,0}-10 \mu \mathrm{m}$.

Comentários. Esta espécie é muito próxima a Terpios manglaris Rützler \& Smith, 1993. Os caracteres diferenciais entre T. belindae e T. manglaris são muito sutis. A característica mais significativa está relacionada com a coloração. Enquanto T. manglaris apresenta cor azul cobalto, conservando-a no material fixado em álcool, $T$. belindae é vermelha, passando à marrom quando preservada na mesma substância (RüTZler \& SMITH 1993). Quanto à morfologia dos tilóstilos e às dimensões dos mesmos também não existe diferença significativa. Um estudo revisivo destas espécies é necessário na tentativa de avaliar os limites de variabilidade inter e intra-especifica.

Distribuição geográfica. Tobago, Golfo do México (RüTZLER \& SмIтн 1993); Brasil: Amapá (no presente estudo).

Distribuição batimétrica. Desde $3 \mathrm{~m}$, Tobago (RüTzler \& Sмітн 1993) até $56 \mathrm{~m}$ (no presente estudo).

\section{Homosclerophorida Plakinidae Schulze, 1880 Plakinastrella onkodes Uliczka, 1929 Figs 29-35}

Plakinastrella onkodes Uliczka, 1929: 60, figs 72-6, pl. 1, fig. 12. (Localidade-tipo: entre S. Thomas e St. John, Caribe); Mothes \& Bastian, 1993: 17, figs 4-6, 37; Diaz \& Van Soest, 1994: 96, fig. 1c; Muricy \& Diaz, 2002: 75.

Para sinônimos adicionais veja ZEA (1987)

Material examinado. MCNPOR $1886\left(03^{\circ} 50^{\prime} 00^{\prime \prime} \mathrm{N}, 50^{\circ} 00^{\prime}\right.$ 07"W), profundidade $81 \mathrm{~m}$, substrato arenoso, 07.XII.1968.

Material estudado para comparação. Plakinastrella onkodes ZMB 4925 Tipo.

Descrição. Fragmento (comprimento $6,0 \mathrm{~cm}$, largura 4,0 $\mathrm{cm}$ e espessura $2,0 \mathrm{~cm}$ ) extremamente delgado, incrustante (Fig. 29). Superfície irregular com reentrâncias e saliências. Ósculos (diâmetro 0,2-0,3 cm) arredondados. Poros não observados. Material preservado de consistência dura e coloração branca acinzentada.

Esqueleto. Ectossoma constituído por uma paliçada de pequenas diodas (Fig. 30). Coanossoma formado por uma reticulação discretamente subisodictial de escleras dos tipos diodas, triodas e caltrops. Escleras: Diodas - diactinas encurvadas,

Revista Brasileira de Zoologia 23 (3): 667-677, setembro, 2006 

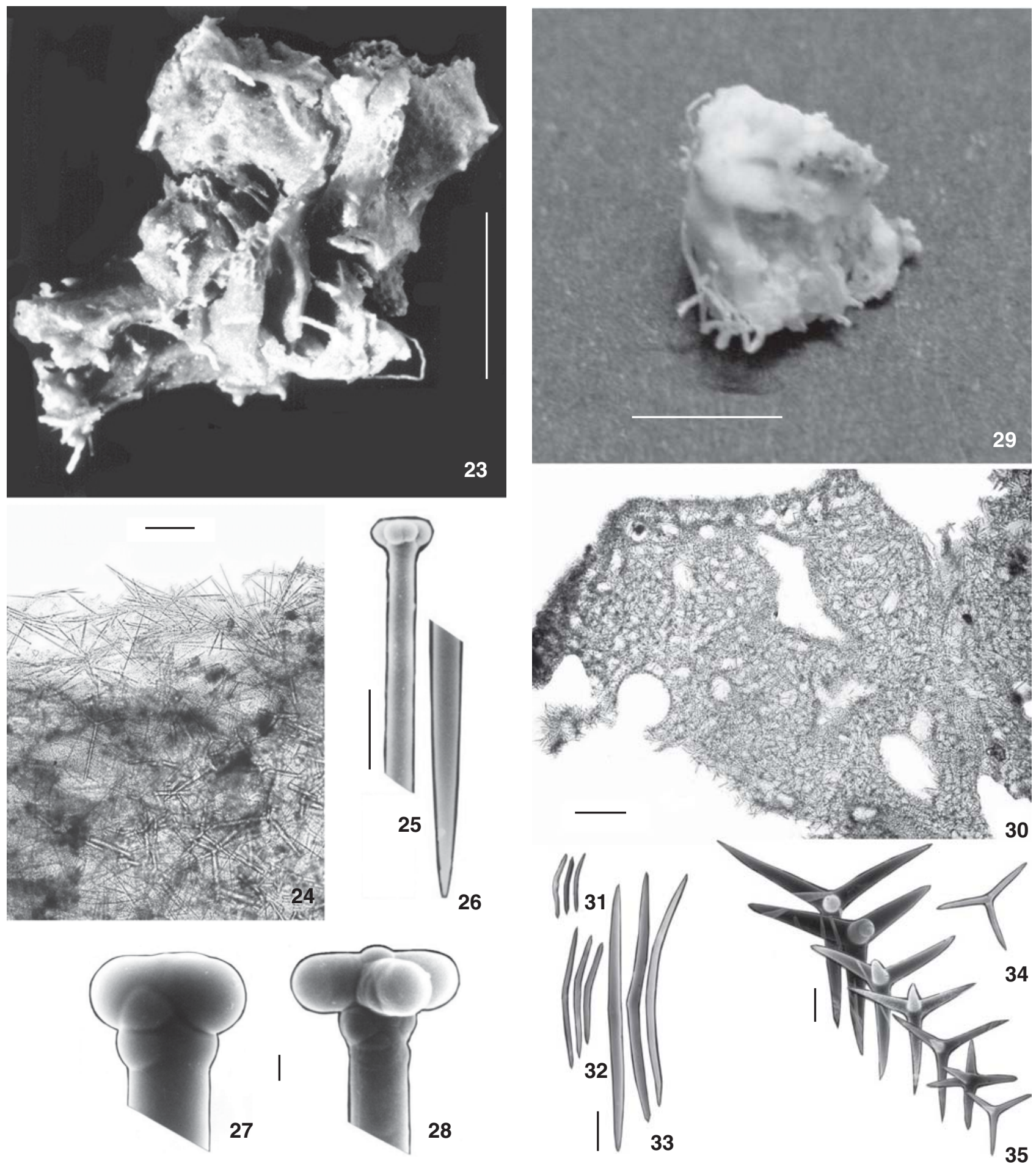

33

Figuras 23-35. (23-28). Terpios belindae: (23) vista do espécime; (24) esqueleto; (25-28) tilóstilo: (25) porção basal; (26) porção apical; (27) cabeça bilobada; (28) cabeça tetralobada; (29-35) Plakinastrella onkodes: (29) vista do espécime; (30) esqueleto; (31) diodas I; (32) diodas II; (33) diodas III; (34) trioda; (35) caltrops. Escalas: (23) $1 \mathrm{~cm}$; (24, 30) $300 \mu \mathrm{m}$; (25-26) $10 \mu \mathrm{m}$; (27-28) $1 \mu \mathrm{m}$; (29) 0,5 cm; (3135) $20 \mu \mathrm{m}$.

Revista Brasileira de Zoologia 23 (3): 667-677, setembro, 2006 
algumas sinuosas, discretamente infladas na região central e/ou paracentral, dimensões: diodas I (Fig. 31) 18,4-24,4-36,8/> 1,0 $\mu \mathrm{m}$, diodas II (Fig. 32), 50-60,7-70/1.0 $\mu \mathrm{m}$, diodas III (Fig. 33) 92,5-136,4-177,5/1,25-4, 4-5,0 $\mu \mathrm{m}$; triodas (Fig. 34) - raras (observadas três escleras), triactinas com eixos delgados, extremidades discretamente encurvada, dimensões dos eixos: comprimento 34,5-39,1 $\mu \mathrm{m}$ e largura junto à base 3,5-6,9 $\mu \mathrm{m}$; caltrops (Fig. 35 ) - tetractinas delgadas a robustas, eixos retos, abruptamente pontiagudos, alguns com extremidade discretamente encurvada, grande variedade de tamanho, dimensões dos eixos: comprimen-

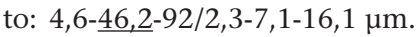

Comentários. Por ser muito sutil a presença de classes de tamanhos diferentes das escleras do tipo caltrops, optou-se por considerar como um gradiente de tamanho.

Distribuição geográfica. Dry Tortugas a Colômbia (Laubenfels 1936, Uliczka 1929, Winterman-Killian \& Killian 1984, ZeA 1987); Brasil: Amapá (no presente estudo); Arquipélago de Fernando de Noronha (Mothes \& Bastian 1993).

Distribuição batimétrica. Desde 12 m, Cartagena, Colômbia (ZeA 1987) até 92 m, Amapá (no presente estudo).

\section{CONSIDERAÇÕES ZOOGEOGRÁFICAS}

O primeiro indício que haveria um substrato disponível para a presença de demospongias no norte do Brasil foi registrado por Ottman (1959) apud Hechtel (1976). Laborel (1969), ao estudar cnidários da costa brasileira, realizou um amplo estudo geográfico, físico, químico e geológico de todas as zonas costeiras do Brasil; entre outras afirmações considerou, para a porção norte da costa, que nos depósitos de argila poderia ocorrer uma rica espongofauna, e que os sedimentos calcários poderiam abrigar, além de esponjas, também briozoários e octocorais. Com base nessas observações, Hechtel (1976) afirmou que esponjas seriam capazes de utilizar esses substratos como verdadeiros "oásis" para sua colonização, entre o Orinoco e o Amazonas.

O conhecimento sobre a riqueza de espécies de poríferos na região do golfão amazônico, na qual a área estudada está inserida, é ainda insignificante para visualizar a existência de uma possível área endêmica. Até o momento foram identificadas 37 espécies, incluindo-se os resultados do presente estudo (Coelho \& Mello-Leitão 1978, Hajdu \& Desqueyroux-Faúndez 1994, Мотнеs et al. 1999, 2004a, b, c, Мотнеs \& Campos 2004, CAmpos et al. 2005). Destas 32 são co-específicas com espécies do Caribe e cinco são novas para a ciência, consideradas aqui provisoriamente endêmicas. A maior parte das espécies já identificadas para a região tem seu registro a partir da plataforma interna, ao contrário das mesmas encontradas no infralitoral do Caribe. Esta profundidade maior é uma forma de adaptação dos organismos, originada pelos efeitos físicos dos rios que desembocam à costa, assegurando assim também uma maior dispersão. Um amplo sucesso de dispersão e adaptação pode ser constatado pela espécie $A$. fulva, com registro setentrional no Caribe e meridional no infralitoral de Bombinhas, Santa Catarina.

\section{AGRADECIMENTOS}

A José A.C. Luna, Departamento de Oceanografia, Universidade Federal de Pernambuco; Rob van Soest, Instituut voor Systematiek en Populatiebiologie, Zoölogisch Museum, Amsterdam pela revisão das identificações; Klaus Rützler, Department of Systematic Biology, Smithsonian Institution, Washington; Francisco Kiss, Departamento de Metalurgia, Universidade Federal do Rio Grande do Sul. Ao CNPq, CAPES e FAPERGS pelo suporte financeiro e concessão de bolsas.

\section{REFERÊNCIAS BIBLIOGRÁFICAS}

Arndt, W. 1927. Kalk- und Kieselschwämme von Curaçao. Bijdragen tot de Dierkunde, Amsterdam, 25: 133-158.

Boury-Esnault, N. 1973. Campagne de la Calypso au large des côtes atlantiques de l'Amérique du Sud (1961-1962). I, 29. Spongiaires. Résultats Scientifiques des Campagnes de la "Calypso", Paris, 10: 263-295.

Bowerbank, J.S. 1858. On the anatomy and physiology of the Spongiadae. Part I: On the spicula. Philosophical Transactions of the Royal Society of London, London, 168: 279-332.

Campos, M.; B. Mothes; R. Eckert \& R.W.M Van Soest. 2005. Haplosclerida (Porifera: Demospongiae) from the coast of Maranhão State, Brazil, Southwestern Atlantic. Zootaxa, Auckland, 963: 1-22.

Coelho, E.P. \& A. Mello-Leitão. 1978. Placospongia carinata e sua ocorrência em costas brasileiras. Avulso, Departamento de Zoologia, Universidade Universidade Federal do Rio de Janeiro, Rio de Janeiro, 29: 1-5.

Díaz, M.C. \& R.W.M. Van Soest. 1994. The Plakiniidae: A Systematic Review, p. 93-109. In: R.W.M. VAN SOEST; T.M.G. VAN Kempen \& J.C. Braekman (Eds). Sponges in Time and Space. Rotterdam, Balkema, 544p.

Duchassaing De Fonbressin, P. \& G. Michelotti. 1864. Spongiaires de la mer Caraibe. Natuurkundige Verhandelingen van de Hollandsch Maatschappij der Wetenschappen, Haarlem (2) 21 (3): 1-124.

ЕкмАN, S.P. 1953. Zoogeography of the sea. London, Sidgwick and Jackson, XIV+417p.

Gray, J.E. 1867. Notes on the arrangement of sponges, with the description of some new genera. Proceedings of Zoological Society of London 1867 (2): 492-558.

Hajdu, E. \& R. Desqueyroux-Faúndez. 1994. A synopsis of South American Mycale (Mycale) (Poecilosclerida, Demospongiae), with the description of three new species and a cladistic analysis of Mycalidae. Revue Suisse de Zoologie, Genebra, 101 (3): 563-600.

Hechtel, G.J. 1965. A systematic study of the Demospongiae of Port Royal, Jamaica. Bulletin Peabody Museum of Natural History, New Haven, 20: 1-94.

Hechtel, G.J. 1976. Zoogeography of Brazilian Marine Demospongiae, p. 237-259. In: F.W. Harrison \& R.R. CoWden (Eds). Aspects of Sponge Biology. New York, 354p.

Hooper, J.N.A. \& R.W.M Van Soest. 2002. Systema Porifera: a

Revista Brasileira de Zoologia 23 (3): 667-677, setembro, 2006 
guide to the classification of sponges. New York, Kluwer Academy, Plenum Publishers, 1708p.

Johnson, M.F. 1971. Some marine sponges of northeast Brazil. Arquivo de Ciências do Mar, Fortaleza, 11 (2): 103-116.

LABOREL, J. 1969. Los peuplements de madreporaires des côtes tropicales du Brésil. Annales de L'Université D'Abidjan, Abidjan, 2 (3): 1-260.

LAMARCK, J.B.P.A. 1816. Histoire naturelle des animaux sans vertébres. Paris, Verdiére, 2: 1-568; 3: 1-586.

LAUbenfels, M.W. DE. 1936. A discussion of the sponge fauna of the Dry Tortugas in particular, and the West Indies in general, with material for a revision of the families and orders of the Porifera. Papers of the Tortugas Laboratory, Washington, 30: 1-225.

Lehnert, H. \& R.W.M. Van Soest. 1996. North Jamaican deep fore-reef sponges. Beaufortia, Amsterdam, 46 (4): 53-81.

Mothes, B. \& M.C.K. DE A. Bastian. 1993. Esponjas do Arquipélago de Fernando de Noronha (Porifera, Demospongiae). Iheringia, Série Zoologia, Porto Alegre, 75: 15-31.

Mothes, B. \& M.A. Campos. 2004. Biemna trisigmata sp. n., a new sponge from the North coast of Brazil (Demospongiae, Poecilosclerida). Zootaxa, Auckland, 639: 1-7.

Mothes, B. \& C.B. LeRner. 1994. Esponjas marinhas do infralitoral de Bombinhas (Santa Catarina, Brasil) com descrição de três espécies novas (Porifera: Calcarea e Demospongiae). Biociências, Porto Alegre, 2 (1): 47-62.

Mothes, B. \& C.B. Lerner. 2001. A new species of Erylus Gray, 1867 (Porifera, Geodiidae) from the southeastern coast of Brazil. Beaufortia, Amsterdam, 51 (4): 83-89.

Mothes, B.; C.B. Lerner. \& C.M.M. Silva. 1999. Revision of Brazilian Erylus (Porifera, Demospongiae) with description of a new species. Memoirs of the Queensland Museum, Brisbane, (44): 369-380.

Mothes, B.; M.A. Campos; C. Lerner \& M.M. Ferreira-Correia. 2004a. Esponjas (Demospongiae, Halichondrida) da costa do Maranhão, Brasil. Iheringia, Série Zoologia, Porto Alegre, 94 (2): 149-154.

Mothes, B.; E. Hajdu; C. Lerner \& R.W.M. Van Soest. 2004b. Two new species (Demospongiae, Poecilosclerida) from the NNE Brazilian continental shelf. In: Pansini, M.; Pronzato, R.; Bavestrello, G. \& Manconi, R. (Eds.). Sponge Science in the New Millennium. Bolletino dei Musei e degli Istituti Biologici della Università di Genova, Genova, 68: 477-482.

Mothes, B.; C.P. SANTOS \& M.A. CAMPOS. 2004c. Timea bioxyasterina sp. n., a new species from the Northeastern coast of Brazil (Demospongiae, Hadromerida). Zootaxa, Auckland. 44 (3): $1-8$.

Mothes-De-Moraes, B. 1978. Esponjas tetraxonidas do litoral sulbrasileiro: II. Material coletado pelo N/Oc. "Prof. W. Besnard" durante o Programa RS. Boletim do Instituto Oceanográfico, São Paulo, 27 (2): 57-78.

Muricy, G. \& M.C. Díaz. 2002. Order Homosclerophorida Dendy, 1905, Family Plakinidae Schulze, 1880, p. 71-84p. In: J.N.A
Hooper \& R.W.M. VAN Soest (Eds). Systema Porifera: a guide to the classification of sponges. New York, vol. 1, XLVIII+1708p.

Muricy, G. \& F. Moraes. 1998. Marine sponges of Pernambuco state, NE Brazil. Revista Brasileira de Oceanografia, São Paulo, 46 (2): 213-217.

Muricy, G.; E. Hajdu; M. Custodio; M. Klatau; C. Russo \& S. Peixinho. 1991. Sponge distribution at Arraial do Cabo, SE Brazil, p. 1183-1195. In: O.T. Magoon; H. Converse; V. TippIE; L.T. Tobin \& D. Clark (Eds). Symposium of coastal and oceanography management. Long Beach, ASCE Publications, vol. 2, 3978p.

Muricy, G.; E. Hajdu; F.V. Araújo \& A.N. Hagler. 1993. Antimicrobial activity of Southwestern Atlantic shallow-water marine sponges (Porifera). Scientia Marina, Barcelona, 57 (4): 427-432.

Nichols, S.A \& P.A.G. Barnes. 2005. A molecular phylogeny and historical biogeography of the marine sponge genus Placospongia (Phylum Porifera) indicate low dispersal capabilities and widespread crypsis. Journal of Experimental Marine Biology and Ecology, Amsterdam. 323: 1-15.

PALLAS, P.S. 1766. Elenchus zoophytorum sistens generum adumbrationes generaliores et specierum cognitarum succintas descriptiones cum selectis auctorum synonymis. The Hague, Van Cleef, 415p.

Pinheiro, U. dos S. \& E. Hajdu. 2001. Shallow-water Aplysina Nardo (Aplysinidae, Verongida, Demospongiae) from the São Sebastião Channel and its environs (Tropical southwestern Atlantic), with the description of a new species and a literature review of the other Brazilian records of the genus. Revista Brasileira de Zoologia, Curitiba, 18 (1): 143-160.

RüTZLer, K. 2002. Family Placospongiidae Gray, 1867, p. 196200. In: J.N.A. HOOPER \& R.W.M. VAN SOEST (Eds). Systema Porifera: a guide to the classification of sponges. New York, vol. 1, XLVIII+1708p.

Rützler, K. \& K.P. Sмith. 1993. The genus Terpios (Suberitidae) and new species in the "Lobiceps" complex. Scientia Marina, Barcelona, 57 (4): 381-393.

SARÀ, M. 1958. Contributo alla conoscenza dei Poriferi del Mar Ligure. Annali del Museo Civico di Storia Naturale di Genova, Genova, 70: 207-244.

Solé-Cava, A.M.; A. Kelecom \& G.J. Kannengiesser. 1981. Study of some sponges (Porifera, Demospongiae) from the infralitoral of Guarapari, Espírito Santo, Brazil. Iheringia, Série Zoologia, Porto Alegre, 60: 125-150.

UliczKa, E. 1929. Die tetraxonen Schwämme Westindiens (auf Grund der Ergebnisse der Reise Kükenthal-Hartmeyer). Zoologische Jahrbücher, Jena, Suppl. 16: 35-62.

VACelet, J. 1959. Répartition générale des éponges et systématique des éponges cornées de la région de Marseille et de quelques stations méditerranéennes. Recueil des Travaux de la Station Marine d'Endoume, Endoume, 26: 39-101.

VAN SOEST, R.W.M. 1978. Marine sponges of Curaçao and other 
Caribbean localities. Part I. Keratosa. Studies on the Fauna of Curaçao and other Caribbean Islands, Amsterdam, 56 (179): 1-94.

VAN SoEST, R.W.M. 1980. Marine sponges of Curaçao and other Caribbean localities. Part II. Haplosclerida. Studies on the Fauna of Curaçao and others Caribbean Islands, Amsterdam, 62 (191): 1-173.

Van Soest, R.W.M. \& N. Stentoft. 1988. Barbados deep water sponges. Studies on the Fauna of Curaçao and others Caribbean Islands, Amsterdam, 70: 1-175.

VANNUCCI, M. 1964. Zoogeografia marinha do Brasil. Boletim do Instituto de Biologia Marinha, São Paulo, 7: 113-121.
Vosmaer, G.C.J. \& J.H. Vernhout. 1902. The Porifera of the Siboga Expedition, I. The genus Placospongia, p. 1-17. In: M. Weber (Ed.). Siboga-Expeditie. Leiden, E.J. Brill, 17p.

WIEDENMAYER, F. 1977. Shallow-water sponges of the western Bahamas. Basel Birkhäuser Verlag, 287p.

Wintermann-Kilian, G. \& E.F. Kilian. 1984. Marine Sponges of the Region of Santa Marta (Colombia) - Part II. Homosclerophorida, Choristida, Spirophorida, Hadromerida, Axinellida, Halichondrida, Poecilosclerida. Studies on Neotropical Fauna and Environment, Alblasserdam. 19 (3): 121-135.

ZEA, S. 1987. Esponjas del Caribe Colombiano. Bogotá, Editorial Catalogo Científico, 286p.

Recebido em 07.X.2005; aceito em 15.VIII.2006.

Revista Brasileira de Zoologia 23 (3): 667-677, setembro, 2006 